\title{
Dog Bite
}

National Cancer Institute

\section{Source}

National Cancer Institute. Dog Bite. NCI Thesaurus. Code C122419.

A wound received from the teeth of a dog. 